\title{
Pulmonary Mucormycosis Presenting as Nonresolving Pneumonia in a Patient With Diabetes Mellitus
}

\author{
Manoj K Panigrahi MD, Rajaram Manju MD, Saka Vinod Kumar MD, and Pampa C Toi MD
}

\section{Introduction}

Zygomycosis refers to a group of disorders caused by filamentous fungi in the class Zygomycetes. This class is subdivided into 2 orders, Mucorales and Entomophthorales, both implicated in human disease. The majority of human infections are caused by fungi in the Mucorales order; hence, the terms zygomycosis and mucormycosis are often used interchangeably. The human population is most frequently affected by the Mucor, Rhizopus, Absidia, and Chlamydoconidia species, with Mucor being the most common. Members of the Mucorales order are saprophytic organisms that are ubiquitous in nature and enter the human body through the respiratory tract or non-intact skin. They mostly affect people with hematological malignancy, hematopoietic stem cell transplantation, prolonged neutropenia, diabetes mellitus, and trauma. Other immunocompromised patients at risk include those using corticosteroids or desferoxamine, undergoing solid organ transplantation or long-term voriconazole prophylaxis, or with iron overload. ${ }^{1,2}$ These fungi are highly vasotropic, with a propensity to cause thrombosis and tissue necrosis, and they are associated with very high mortality.

Although most fungal infections, including mucormycosis, are acquired via inhalation, endobronchial manifestation is rare, and the usual presentation is either pulmonary or systemic disease. Among the endobronchial fungal infections, Aspergillus subspecies are the most common, and endobronchial mucormycosis (EBM) is still uncom-

\footnotetext{
Dr Panigrahi is affiliated with the All India Institute of Medical Sciences, Odisha, India. Drs Manju and Vinod Kumar are affiliated with the Department of Pulmonary Medicine, and Dr Toi is affiliated with the Department of Pathology, Jawaharlal Institute of Postgraduate Medical Education and Research, Puducherry, India.

The authors have disclosed no conflicts of interest.

Correspondence: Manoj K Panigrahi MD, Department of Pulmonary Medicine, All India Institute of Medical Sciences, Bhubaneswar, 751019 Odisha, India. E-mail: manoj_kp99@rediffmail.com.
}

DOI: $10.4187 /$ respcare. 03205 mon. The majority of endobronchial fungal infections occur in association with systemic dissemination in immunocompromised hosts. ${ }^{3}$ Herein, we report a case of isolated pulmonary mucormycosis with endobronchial involvement presenting as nonresolving pneumonia in a diabetic patient who was successfully managed with medical therapy.

\section{Case Summary}

A 58-y-old male was referred for an inadequately responding cavitary infiltrate in the right lung after receiving $10 \mathrm{~d}$ of intravenous levofloxacin and clindamycin. He presented with 1 month of fever, productive cough with purulent sputum, and breathlessness on exertion. He worked as security personnel, was a nonsmoker, and had never traveled outside his place of residence. He had diabetes mellitus for last $18 \mathrm{y}$ and was receiving oral glucoselowering agents. He denied any intake of corticosteroids or immunosuppressive agents in the past. On examination, he was awake and alert but appeared unwell. He had a heart rate of 109 beats/min, a blood pressure of $126 / 80 \mathrm{~mm} \mathrm{Hg}$, a breathing frequency of 27 breaths/min, and a temperature of $38.8^{\circ} \mathrm{C}$. His $\mathrm{S}_{\mathrm{pO}_{2}}$ was $95 \%$ on room air. General examination was unremarkable. Chest auscultation revealed decreased breath sounds and scanty crackles in the right mammary area. Examination of other systems was normal. The hemoglobin level was $12.2 \mathrm{~g} / \mathrm{dL}$, the white blood cell count was 7,100 cells/ $\mu \mathrm{L}$ (polymorphs, $60 \%$; lymphocyte, $37 \%$; eosinophils, 3\%), and the platelet count was 210,000 cells $/ \mu \mathrm{L}$. A blood metabolic panel provides the following results: urea, $22 \mathrm{mg} / \mathrm{dL}$; creatinine, $1.2 \mathrm{mg}$ / $\mathrm{dL}$; total bilirubin, $0.9 \mathrm{mg} / \mathrm{dL}$; albumin, $4.3 \mathrm{~g} / \mathrm{dL}$; aspartate, aminotransferase, $83 \mathrm{IU} / \mathrm{L}$; alanine aminotransferase, $72 \mathrm{IU} / \mathrm{L}$; alkaline phosphates, $60 \mathrm{IU} / \mathrm{L}$; sodium, $122 \mathrm{mEq} / \mathrm{L}$; and potassium, $4.4 \mathrm{mEq} / \mathrm{L}$. Fasting blood glucose on admission was $191 \mathrm{mg} / \mathrm{dL}$, with glycated hemoglobin of $7.5 \%$. Chest radiograph on admission showed a large cavity in the right parahilar lung field (Fig. 1A). Repeated sputum smears for acid-fast bacilli were negative. Serology for human immunodeficiency virus and hepatitis B antigen was negative. Necrotizing pneumonia was diagnosed, and empirical intravenous ceftriaxone, ceftazidime, and met- 


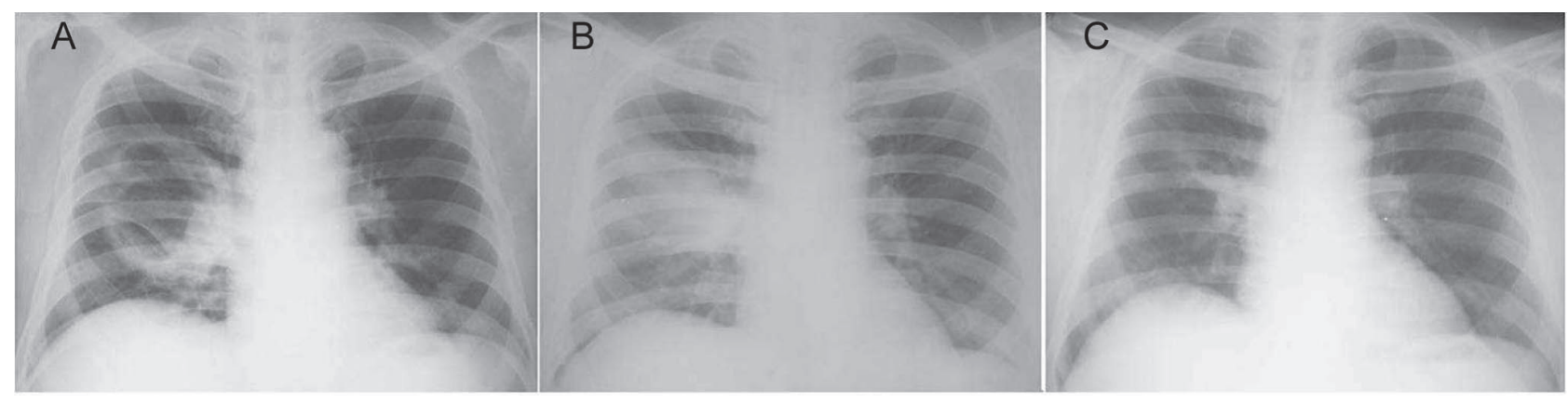

Fig. 1. Serial chest radiographs of the subject in different stages of resolution. A: On admission, showing a large cavity in the right parahilar lung field with normal-looking surrounding parenchyma. B: Ten weeks after the initial presentation, showing unresolved consolidation in the right mid and upper lung fields. C: Following completion of antifungal therapy, demonstrating near-complete resolution of the radiopacity.

ronidazole were started. Expectorated sputum culture showed growth of Escherichia coli sensitive to ceftazidime and amikacin. Blood culture for bacteria was sterile. The antimicrobial regimen remained unchanged, clinical symptoms gradually improved, and a trend of radiographic resolution was seen. The patient was discharged after 3 weeks of treatment.

Six weeks later, he presented again with new-onset cough and yellow sputum periodically admixed with blood. He had no fever, chest pain, headache, or rhinitis symptoms. He looked well, and all vital parameters were normal. Repeat chest radiograph showed inadequate resolution of the previous opacity (see Fig. 1B). All routine blood tests were normal except for mild hyperglycemia. In view of the fresh hemoptysis, nonresolving pneumonia resulting from an endobronchial lesion was considered, and flexible bronchoscopy was performed. Bronchoscopy revealed a whitish mass completely occluding the anterior segment of right upper lobe bronchus, with band-like extension to the right main bronchus and trachea (Fig. 2). Endobronchial biopsy showed aseptate fungal hyphae, right-angle branching, and tissue infiltration of neutrophils (Fig. 3). Bronchial wash fungal culture was sterile, and sputum mycobacterial culture was negative.

Our patient received intravenous amphotericin B at $60 \mathrm{mg} / \mathrm{d}(1 \mathrm{mg} / \mathrm{kg})$ for $12 \mathrm{~d}$. Treatment was switched to posaconazole on the 13th day, honoring his request for discharge and refusal of amphotericin due to mild infusion-related toxicity. He managed to take posaconazole at $400 \mathrm{mg}$ twice daily for the next 4 weeks and then discontinued due to cost. Hemogram and renal function remained normal during the treatment. Clinical and radiological improvement was remarkable (see Fig. 1C). Hyperglycemia was controlled initially with insulin, followed by oral hypoglycemic agents. Repeat bronchoscopy performed 2 weeks after discontinuation of posaconazole therapy confirmed clearing of the endobronchial mass.

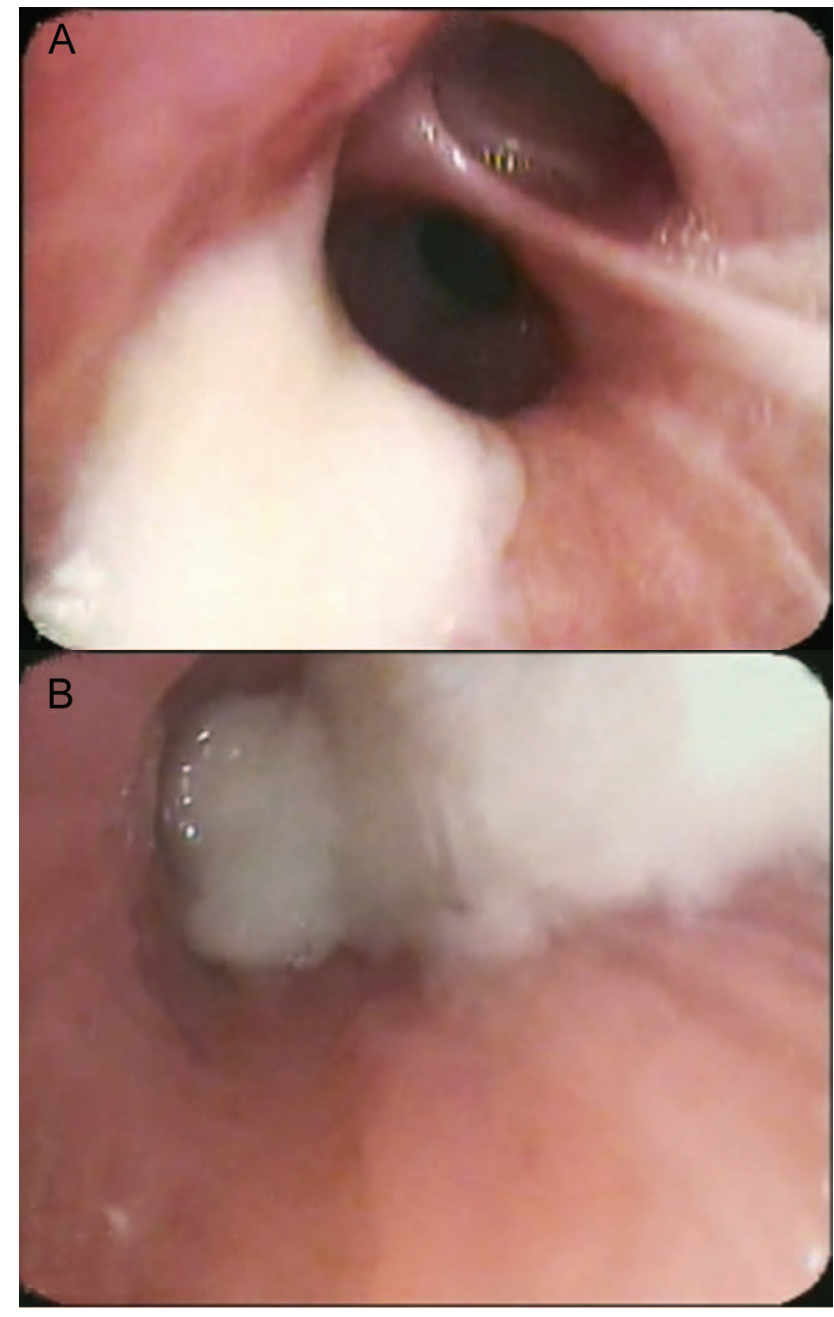

Fig. 2. A: Bronchoscopic image of the right upper lobe showing normal apical and posterior segments and a whitish mass completely occluding the anterior segment. B: Flexible bronchoscopy demonstrating a whitish endobronchial mass with a thick bandlike proximal extension in the anterior segment of the right upper lobe. 


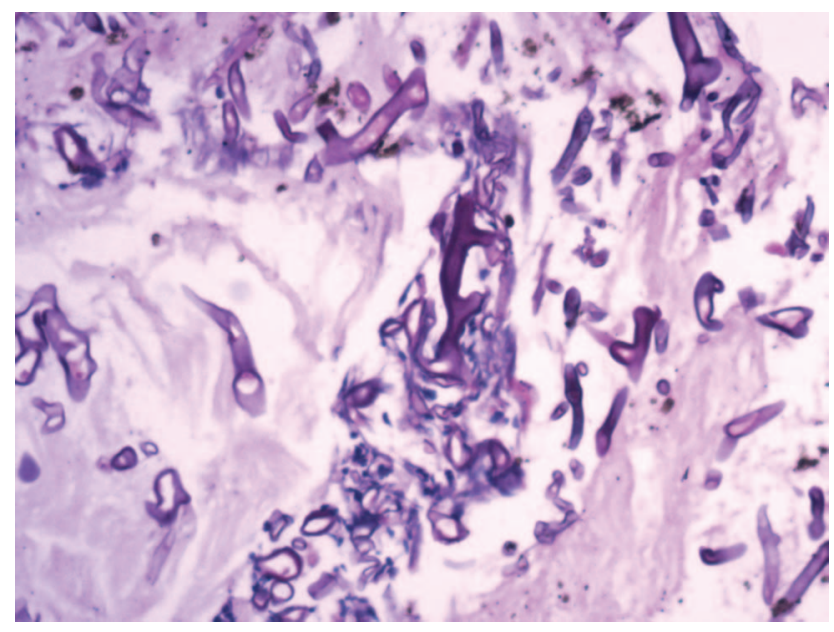

Fig. 3. Endobronchial biopsy demonstrating colonies of broadbased, aseptate fungal hyphae with right-angle branching, consistent with mucormycosis (periodic acid-Schiff stain, magnification $\times 20$ ).

\section{Discussion}

Mucormycosis has emerged as the third most common invasive fungal infection after candidiasis and aspergillosis in patients with hematological malignancy and hematopoietic stem cell transplantation. Based on the anatomic site involved, mucormycosis can be one of several forms, such as rhinocerebral, pulmonary, cutaneous, gastrointestinal, disseminated, and uncommon presentations that include endocarditis, osteomyelitis, peritonitis, and renal infection. The underlying predisposing conditions can influence clinical presentation and outcome. Pulmonary infection is the most common form of mucormycosis recognized in patients with hematological malignancy and remains the second most common presentation after rhinocerebral infection in diabetic patients. ${ }^{1}$ Uncontrolled diabetes, particularly diabetic ketoacidosis, predisposes patients to the development of mucormycosis; however, cases have been described even in patients with well controlled diabetes mellitus. ${ }^{4}$ These fungi have a ketone reductase enzyme that allows them to thrive in a high-glucose environment. Increased iron uptake by the fungi stimulates fungal growth and may lead to clinical infection. Diabetic individuals have increased serum iron because of impaired transferrin binding, which may contribute to their increased risk of infection. ${ }^{3}$

Patients usually present with prolonged high-grade fever that is unresponsive to broad-spectrum antibiotics. Nonproductive cough is a common symptom, whereas hemoptysis, pleuritic chest pain, and dyspnea are less common. ${ }^{1,2}$ Endobronchial lesions are found in one third of patients with pulmonary mucormycosis. If endobronchial involvement occurs, the large bronchi are mainly affected, with a high mortality rate. Patients with EBM present mainly with dyspnea and hemoptysis. EBM occasionally leads to obstruction of major airways, resulting in lung collapse or invasion of major pulmonary blood vessels, leading to fatal hemoptysis. On bronchoscopy, this lesion usually presents as a mass or grayish-white fibrinous plug obstructing the bronchus. Other manifestations of EBM are sloughing of mucosa, granular lesions, ulcers, stenosis, and pseudomembrane formation. The majority of EBM cases have been described in patients with diabetes mellitus. ${ }^{3}$ Furthermore, pulmonary mucormycosis tends to present a more subacute and less fulminant course in these patients. ${ }^{2}$ Our patient's initial presentation was similar to an acute bacterial lung infection that partially responded to antibiotic therapy, and he subsequently presented with hemoptysis, a common feature in EBM.

The usual radiographic findings of pulmonary mucormycosis include infiltration, consolidation, nodules, cavitations, atelectasis, effusion, and hilar or mediastinal lymphadenopathy. Computed tomography scan results of multiple lung nodules ( $\geq 10$ ), pleural effusion, and reversed halo signs are found to be better predictors of pulmonary mucormycosis than invasive aspergillosis in patients with hematological malignancy. Concomitant sinusitis and voriconazole prophylaxis are significantly associated with development of pulmonary mucormycosis. ${ }^{2,5}$

The clinical and radiographic findings of pulmonary mucormycosis are nonspecific and often indistinguishable from those of pulmonary aspergillosis. Nonetheless, differentiating between the two is of paramount importance, as it has significant management implications because antifungals such as voriconazole and caspofungin, which are active against Aspergillus subspecies, are ineffective in mucormycosis. Moreover, prophylaxis with these drugs has been associated with development of mucormycosis. Considering the fact that $<50 \%$ of cases are diagnosed antemortem, a high index of clinical suspicion is required for timely diagnosis of mucormycosis. The diagnosis is usually made by histopathological demonstration of tissue invasion by the characteristic hyphae or by isolation of Zygomycetes species. In tissue specimens, Zygomycetes appears as broad aseptate hyphae with right-angle branching, whereas Aspergillus species appear as septate and thinner hyphae with acute-angle branching. Tissue infiltration with neutrophils (in non-neutropenic hosts) and necrosis with invasion of blood vessels with thrombosis are quite common in mucormycosis. Biopsy is more yielding than cultures, and many specimens, including blood, sputum, gastric fluid, and nose swabs, are difficult to culture and often have no diagnostic value. Sputum cultures are not reliable indicators of invasive mucormycosis, as some Zygomycetes species often colonize the respiratory tract. 
In addition, nonculture-based diagnostic methods, such as fungal antigen detection and molecular diagnosis using polymerase chain reaction, remain investigational, and no assay with sufficient sensitivity or specificity has been identified. $^{2}$

There are several causes for nonresolving pneumonia that include both infectious and noninfectious pathologies. Poor patient compliance, improper dosing, drug resistance, or development of a complication is the usual reason for nonresolution in patients with infections. Occasionally, infection with uncommon pathogens (such as mycobacteria and fungus) or actinomycosis can lead to nonresolving pneumonia. Endemic fungal infections, such as blastomycosis, histoplasmosis, and coccidioidomycosis, are more commonly described etiologies of nonresolving pneumonia than mucormycosis. ${ }^{6}$ One may suspect pulmonary mucormycosis in susceptible hosts such as immunocompromised and neutropenic patients; however, diagnosing isolated pulmonary mucormycosis in otherwise healthy diabetic individuals is challenging because community-acquired bacterial infections and pulmonary tuberculosis are the more common respiratory infections in such patients. ${ }^{7}$ Furthermore, secondary bacterial infection may alter the clinical and radiological presentation of pulmonary mucormycosis, thereby delaying the diagnosis further. In our patient, the initial radiographic improvement represents the resolution of secondary bacterial infection following antibiotic therapy.

The current management of pulmonary mucormycosis in relatively immunocompetent patients, such as those with diabetes or trauma, is largely driven by the treatment experience gained from immunocompromised and neutropenic patients. The ideal antifungal regimen and duration of therapy in non-neutropenic patients have yet to be determined. Treatment with antifungal agents in combination with surgical debridement is considered as the standard of therapy in invasive mucormycosis. Amphotericin remains the only approved drug for treatment of mucormycosis. Early initiation of amphotericin therapy even empirically has a profound impact on survival. Posaconazole, an orally active newer triazole, has recently shown promising results when used as salvage therapy in mucormycosis. The usual reasons for switching from amphotericin to posaconazole are drug toxicity or failure and discharge purposes. The duration of treatment depends upon the response and control of underlying risk factors. Most investigators have reported improvement within 2 weeks of posaconazole therapy. In a study of 24 subjects for whom posaconazole was used as salvage therapy, the duration varied from 8 to $1,004 \mathrm{~d}$, with a median duration of $182 \mathrm{~d}$. Posaconazole at $200 \mathrm{mg} 4$ times per day or $400 \mathrm{mg}$ twice per day has shown favorable response rates of $60 \%$ and $79 \%$, respectively. ${ }^{8}$ In a retrospective series of invasive mucormycosis, posaconazole therapy was successful in $60 \%$ of subjects with diabetes mellitus, and its overall success rate was $65 \%$ in those with pulmonary mucormycosis. ${ }^{9}$

Despite motivation and pursuance, our patient could not continue posaconazole beyond 4 weeks because of cost. However, we ensured macroscopic clearance of the mass in repeat bronchoscopy 2 weeks after discontinuation of posaconazole, and he did not develop any symptoms of relapse until 1 month ago. This re-emphasizes the need to eliminate the underlying risk factor and also suggests that, if effective glycemic control is maintained, even a short duration of posaconazole therapy may be sufficient to ensure cure and prevent relapse of mucormycosis in patients with diabetes. Strict glycemic control will be the key to prevent any future relapse in our subject. Further studies are needed to elucidate the ideal duration of posaconazole therapy for mucormycosis in diabetic individuals. The available clinical evidence is not sufficient to support the use of combined antifungal therapy.

\section{Teaching Points}

- Physicians should consider mucormycosis as a possibility in evaluation of unresolved pneumonia in patients with diabetes mellitus.

- EBM can present as a secondary bacterial infection that may alter the clinical and radiological presentation, thereby delaying the diagnosis of pulmonary mucormycosis.

- A low threshold should be maintained for performing bronchoscopy in diabetic patients for early diagnosis of EBM.

- Posaconazole is an effective salvage therapy for management of pulmonary mucormycosis in diabetic patients.

\section{REFERENCES}

1. Petrikkos G, Skiada A, Lortholary O, Roilides E, Walsh TJ, Kontoyiannis DP. Epidemiology and clinical manifestations of mucormycosis. Clin Infect Dis 2012;54(Suppl 1):S23-S34.

2. Chamilos G, Kontoyiannis DP. Aspergillus, Candida, and other opportunistic mold infections of the lung. In: Fishman AP, Elias JA, Fishman JA, Grippi MA, Senior RM, Pack AI, editors. Fishman's pulmonary diseases and disorders, 4th edition, volume 2. New York: McGraw-Hill; 2008;2291-2326.

3. Karnak D, Avery RK, Gildea TR, Sahoo D, Mehta AC. Endobronchial fungal disease: an under-recognized entity. Respiration 2007; 74(1):88-104.

4. Di Carlo P, Cabibi D, La Rocca AM, De Luca D, La Licata F, Sacco E. Post-bronchoscopy fatal endobronchial hemorrhage in a woman with bronchopulmonary mucormycosis: a case report. J Med Case Rep 2010;4:398.

5. Chamilos G, Marom EM, Lewis RE, Lionakis MS, Kontoyiannis DP. Predictors of pulmonary zygomycosis versus invasive pulmo- 


\section{Pulmonary Mucormycosis Presenting as Nonresolving Pneumonia}

nary aspergillosis in patients with cancer. Clin Infect Dis 2005;41(1): 60-66.

6. Weyers CM, Leeper KV. Nonresolving pneumonia. Clin Chest Med 2005;26(1):143-158.

7. Ljubic S, Balachandran A, Pavlic-Renar I, Barada A, Metelko Z. Pulmonary infections in diabetes mellitus. Diabetol Croat 2004;33(4): 115-124.
8. Greenberg RN, Mullane K, van Burik JA, Raad I, Abzug MJ, Anstead G, et al. Posaconazole as salvage therapy for zygomycosis. Antimicrob Agents Chemother 2006;50(1):126-133.

9. van Burik JA, Hare RS, Solomon HF, Corrado ML, Kontoyiannis DP. Posaconazole is effective as salvage therapy in zygomycosis: a retrospective summary of 91 cases. Clin Infect Dis 2006;42(7):e61e65. 